\title{
Remembering Places in Space: A Human Analog Study of the Morris Water Maze
}

\author{
Sylvia Fitting, Gary L. Allen, and Douglas H. Wedell \\ Department of Psychology, University of South Carolina, \\ Columbia, SC 29208 USA \\ fitting@sc.edu
}

\begin{abstract}
We conducted a human analog study of the Morris Water Maze, with individuals indicating a remembered location in a $3 \mathrm{~m}$ diameter arena over different intervals of time and with different memory loads. The primary focus of the study was to test a theory of how varying cue location and number of cues affects memory for spatial location. As expected, memory performance as measured by proximity to the actual location was negatively affected by increasing memory load and delay interval and decreasing number of cues. As memory performance decremented, bias effects increased and were in accordance with the cue-based memory model described by Fitting, Wedell and Allen (2005). Specifically, remembered locations were biased toward the nearest cue and error decreased with more cues. These results demonstrate that localization processes that apply to small two-dimensional task fields may generalize to a larger traversable task field.
\end{abstract}

Keywords: Categorical coding, environmental cues, Morris Water Maze, spatial cognition, place memory.

\section{Introduction}

Remembering where objects are located is a common and adaptive activity across species. Several variations on this task may arise from variations in key environmental constraints. For example, the environmental space may be small and two dimensional, such as when remembering a location on the surface of one's computer monitor; moderately large and traversable, such as when remembering where one put one's keys in a room; or much grander in scale and scope, such as when one remembers the location of a residence in the countryside. In addition to the size variable, shape of the environment is a critical factor. For example, several researchers have shown how geometric facets of the environment guide memory for location (Cheng, 1986; Hermer \& Spelke, 1994), with some positing that geometric coding of spatial location may constitute a primitive module for processing this information (Gallistel, 1990). The existence of clearly defined paths is another shape constraint likely to influence memory for location, as numerous studies have documented route specific learning (Siegel \& White, 1979). In addition to size and shape, the surface features of the environment may be critical. Such surface features may serve as proximal or distal cues for coding memory for location (Egan, 1979). 
Clearly it is easier to find one's keys if one remembers placing them next to the lamp (a proximal cue). Or in a large-scale space, one may use the distal cues of surrounding buildings to get a bearing on the location of one's car in a parking lot.

\subsection{Memory for Place}

In general, the psychological literature posits three distinct varieties of spatial learning and memory, including motor learning, association learning and place learning (Morris \& Parslow, 2004; Nadel, 1990; Newcombe \& Huttenlocher, 2000). The focus of the present investigation is on place learning, which is distinguished from the other two varieties of spatial memory by a number of characteristics. Place learning involves acquiring memory for spatial locations independent of specific behaviors associated with those locations (Fenton, Arolfo, Nerad, \& Bures, 1994; Hebb, 1949; Nadel, 1990; Newcombe \& Huttenlocher, 2000; Overman, Pate, Moore, \& Peuster, 1996). This type of memory results from the implicit computation of spatial interrelations between distal cues and to-be-remembered locations within a geometric field (O'Keefe \& Nadel, 1978; Tolman, 1948). Because orientation is based on external cues, place memory is said to involve an allocentric frame of reference. It features the concept of a geometric field that embeds spatial relations among objects and so is also described as involving a coordinate frame of reference (Newcombe \& Huttenlocher, 2000).

Because a geometrical coordinate frame of reference is critical to understanding place learning, researchers have developed a variety of methods to isolate this factor for study. In the animal literature, an important aid in this regard was the development of the Water Maze task by Morris and colleagues (Morris, 1981; Morris, Garrud, Rawlins, \& O'Keefe, 1982). In this task, the rat must swim to a hidden platform located in a circular basin that is surrounded by curtains, composing the walls of the environment. Because the spatial field is undifferentiated, the rat cannot use proximal cues within the search space. Furthermore, because the rat's entry point varies across trials, the rat cannot use a simple sequence of motor responses (such as swim $45^{\circ}$ to the left for 1 meter). Instead, the presence of distal cues on the curtains affords the rat a way to map the platform's location within this environment in a way that takes advantage of stable configural relationships.

While many researchers have utilized an analog to the Water Maze for studying spatial memory in humans (Astur, Ortiz, \& Sutherland, 1998; Astur, Taylor, Mamelak, Philpott, \& Sutherland, 2002; Jacobs, Thomas, Laurance, \& Nadel, 1998; Lehnung, Leplow, Ekroll, Herzog, Mehdorn, \& Ferstl, 2003; Leplow, Lehnung, Pohl, Herzog, Ferstl, \& Mehdorn, 2003; Overman et al., 1996; Parslow, Morris, Fleminger, Rahman, Abrahams, \& Recce, 2005), several alternative procedures have been developed. One extensively studied paradigm has been to have human subjects remember locations within a small two-dimensional field. For example, Huttenlocher, Hedges and Duncan (1991) asked participants to reproduce the location of a dot in a circular region when no explicit external landmark cues were available. Results indicated that stationary observers tended to impose an implicit polar coordinate system on a circular task field and use geometrically based categories to aid in remembering. The focus of these studies has generally been on the bias in location resulting from the categorical coding of spatial location. Although memory for 
location in these tasks is generally very good, the systematic biases typically observed imply humans resolve uncertainty of location by moving toward the prototypical location for the appropriate quadrant or category.

\subsection{Comparison of Procedures}

Because the Morris Water Maze and the dot location task are both used to study place memory, it is instructive to examine similarities and differences between these tasks and corresponding findings. First, the Morris Water Maze task provides evidence for the use of axes extending from visible peripheral cues to organize the field (Morris et al., 1982). In contrast, results from the dot location task are explained in terms of geometric coding, with peripheral cues typically absent in these studies (Huttenlocher et al., 1991). In support of a cue-independent coordinate coding of place, Fitting (2005) demonstrated that including 1 or 3 peripheral cues in the usual dot location task had no effect whatsoever on remembered location. Thus, while the Morris Water Maze has typically been used to study cue-dependent coordinate memory, the dot location task has typically been used to study cue independent coordinate memory.

This difference in the cue dependency of the representations likely arises from procedural differences in the subject's orientation to the task field across these tasks. Whereas the Water Maze procedure includes varying the entry point to the maze so the initial viewer-based information is not diagnostic, the dot location task fixes the orientation of the viewer to the task field so that viewer-based information may be used and peripheral cues are not critical. Fitting et al. (2005) explored the effect of dynamically varying orientation for the dot location task by including peripheral cues and rotating the task field on the majority of trials. Results indicated that in this dynamic environment, subjects used cues to define category prototypes that biased memory for spatial locations. This result held even for those trials during which the task field was not rotated so that peripheral cues could have been ignored.

Another difference between the tasks lies in the time course of remembering. In the spatial memory task used by Huttenlocher et al. (1991), participants reproduce the location of a dot in a circular region a few seconds after the dot disappears from the display. Thus, this task focuses on spatial coding in short-term memory. In contrast, the classic Morris Water Maze procedure examines place memory across days, a long-term memory task (Morris et al., 1982). The difference in the time course of the tasks raises the possibility that different memory mechanisms may apply to the coordinate-based memory systems for these tasks.

The underlying differences in delay intervals across tasks reflect differences in the research questions these tasks have been used to pursue. Those using the dot location task have been primarily interested in how categorical coding may bias spatial memory (Fitting et al., 2005; Huttenlocher et al., 1991; Huttenlocher, Hedges, Corrigan, \& Crawford, 2004; Wedell, Fitting, \& Allen, in press). Short memory durations have been used in this task so that the same subject may be tested on many different locations within the task field, providing a rich data base for modeling the bias in memory. In the category-adjustment model of Huttenlocher et al. (1991), spatial properties of a stimulus specifying its location are represented at two levels, fine-grain and categorical. Categorical coding is posited as the robust product of a 
relatively rapid process in which location is remembered in terms of its being within a particular categorical region of the response space. A central or salient location within this category acts as a prototype, biasing remembered locations toward it. In the specific task of a stationary observer remembering a location within a circular field, observers appear to impose implicit horizontal and vertical axes on the circle, thus creating quadrants that function as categories, with centrally located points within these quadrants serving as prototypes. Although use of these categorical codes may bias estimates, they may also serve the adaptive purpose of reducing overall error in estimation (Huttenlocher et al., 1991). Fine-grain coding is posited as the product of the process of remembering a location in terms of a geometric coordinate system imposed upon the response field. When the task field is circular, this coordinate system is typically defined by polar coordinates. Fine-grain coding yields metric accuracy, but it may also be more fragile than categorical coding, resulting in a greater reliance on categorical coding with increased memory demands (Haun, Allen, $\&$ Wedell, 2005).

In contrast to the goal of studying categorical effects on coordinate memory, the goals addressed by place memory studies using the Morris Water Maze have typically been related to understanding the neurobiology of place learning as distinguished from other varieties of spatial memory. Using these procedures, evidence from neuropsychological and neuroimaging studies in animal and human research indicates that hippocampal areas are uniquely active in place learning (Grön, Wunderlich, Spitzer, Tomczak, \& Riepe, 2000; Jarrard, 1993; Morris \& Parslow, 2004; O’Keefe \& Nadel, 1978, Sutherland \& Rudy, 1987). In addition, a number of studies have shown that damage to the hippocampus and related structures in rodents and humans causes impairment in place learning (Astur et al., 1998; Astur et al., 2002; Devan, Goad, \& Petri, 1996; Jarrard, 1993; Parslow et al., 2005; Sutherland \& Rudy, 1987). Furthermore, psychophysiological studies have shown that principal neurons of the CA1, CA3, and dentate gyrus subfields of the rat hippocampus fire selectively when the animal occupies certain locations in an environment (O'Keefe \& Nadel, 1978). Such place cells appear to participate in a distributed and nontopographic map-like representation of the spatial environment (Knierim \& Rao, 2003; O’Keefe \& Nadel, 1978).

\subsection{Human Analog of the Morris Water Maze}

We believe that while both the Water Maze and dot location paradigms have provided extremely useful evidence relevant to spatial memory for place, additional insights may be gained by a line of research that combines aspects of these paradigms. The study we describe here builds on a study we conducted that utilized dynamically changing orientation in the dot location task (Fitting et al., 2005). That study showed that the inclusion of rotation trials resulted in participants using a cue-based representation of stimulus locations so that cue locations explained the pattern of bias obtained in memory estimates. The current study addresses two shortcomings in generalizing results of the previous study to procedures more akin to those used in the Morris Water Maze. First, there is the question of whether results from a small space, two-dimensional location task generalize to memory for place in a larger-space environment that is physically traversable. A recent study by Haun et al. (2005) is relevant to this point because they demonstrated that spatial bias pattern in incline estimation and azimuth 
estimation tasks within a larger-scale environment show similar biases found in the small-scale two dimensional dot location task. Related to this question is whether the use of cues displayed in the small-scale environment that give rise to a more allocentricbased place memory will be similar to cue use when cues are much more peripheral and must be encoded relative to one's location in space, as in the classic Morris Water Maze. A second major point explored in the current study is whether cues may be used as prototypes when location must be remembered for not a matter of seconds, but rather for minutes or even for weeks, durations more consistent with investigations using the classic Morris Water Maze procedure.

To address these questions, we developed a human analog of the Morris Water Maze which consisted of a circular arena $3 \mathrm{~m}$ in diameter and $3 \mathrm{~m}$ tall. The walls of the arena consisted of black curtains that reached to the floor, with nine different entry points. The floor of the arena was covered in wood chips so there were no discernable proximal cues to use in locating objects. The roof of the arena was undifferentiated as well. Cues were hung on the walls of the arena to provide external reference points for coding the location of objects within the arena.

Note that other researchers have developed human analogs of the Water Maze (Astur et al., 1998; Astur et al., 2002; Jacobs et al., 1998; Lehnung et al., 2003; Leplow et al., 2003; Parslow et al., 2005). One way that our research differs from this previous research is that our primary focus was on cue-based bias, as predicted by our work from the dot location studies. In this regard, the main independent variable was the number (and corresponding locations) of cues. However, because of the extreme differences between the dot location task and the arena task, we included two additional elements designed to affect memory performance. The first of these we call memory load, and it was manipulated between subjects. In the low-load condition, participants were given only one object location to remember. In the high-load condition, they were given three object locations to remember. We predicted better memory when memory load was low. This prediction follows from short-term studies in which having to remember more object locations results in poorer memory for location (Dent \& Smyth, 2005). Additionally, it follows from the idea that holding multiple locations in memory could serve to interfere with memory for any given target and hence lead to poorer memory performance. The second manipulation was delay interval, which we manipulated within subjects. The first interval consisted of 10 to 15 minutes after initial encoding, with the delay interval filled with a spatial memory task. The second interval was one week after the first testing, and the third interval was one week after the second testing. As in any memory study, we predicted poorer memory performance with increased delay interval.

There were two main reasons to include the load and delay interval variables. The first of these was simply to document the pattern of learning and forgetting relevant to these variables in the human analog study. Thus, we wanted to see how much one forgets over time and how difficult it is to remember a location when having to remember two other locations in the same space. The second and more important reason for manipulating these variables was to create conditions under which substantial forgetting would take place. Forgetting was necessary because observing bias effects typically requires there to be some disruption of fine-grain memory. With perfect remembering, there would be no way to test hypotheses regarding bias. 


\section{Two Cues $\quad$ Four Cues}

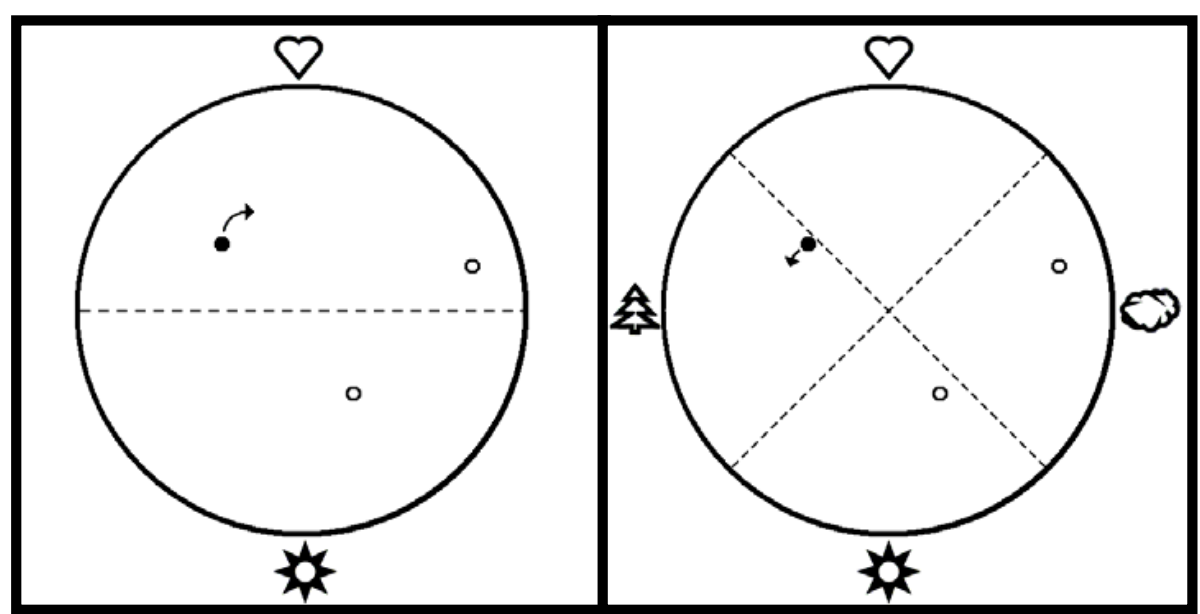

Fig. 1. Schematic illustration of target and cue locations in the arena along with predictions of bias. Dashed lines represent boundaries based on cue determined prototypes. The filled dot shows the target object location, and the open dots show the additional object locations used in the high-load condition. Size of cues and object locations are not to scale.

The cue-based fuzzy-boundary model developed by Fitting et al. (2005) was used to generate predictions regarding the effect of cue manipulation. To illustrate these predictions we present the critical features of the experimental design in Fig. 1. In the two-cue condition (left panel), a heart was located at one end and a sun was located at the other. In the four-cue condition, a tree and a cloud were added at the locations shown. We generated three predictions based on our cue-based fuzzy-boundary model (Fitting et al., 2005). First, we predicted that if cues determine category prototypes, then a bias toward the most proximal cue should be observed for misremembered locations. This prediction is illustrated in Fig. 1 by the arrows, with bias toward the heart in the two-cue condition and toward the tree in the four-cue condition. Thus bias is predicted to be in opposite directions for the two conditions. Note that we only applied this prediction to angular bias, as previous work with the dot location task showed large cue-based effects on angular bias but not on radial bias (Fitting, 2005). Second, our cue-based fuzzy-boundary model predicted that absolute error should decrease with more cues. This is because bias is a key contributor to error, and bias generally increases with distance from the nearest prototypes. With more cues creating more prototypes, the distances to adjacent prototypes tend to decrease, and hence error should correspondingly decrease (indicated in Fig. 1 by differences in the lengths of the arrows in the left and right Panel). Third, we expected bias to be greatest when absolute error was greatest. Thus, we expected bias to be more likely in the long delay-interval conditions and with high load.

A final issue examined in this study was the correlation between measures of place learning and memory on the one hand and measures from tests of small-scale spatial 
abilities on the other hand. Currently, the relation between place memory and smallscale spatial tasks is simply unclear. We reasoned that if place learning and memory is a unique variety of spatial memory, it should not be closely related to the ability to do spatial tasks that require coding spatial relations in terms of a viewer's axes or in terms of an object's intrinsic axes. However, place memory might be related to the ability to remember the locations of objects in a task field, even when that field is small and viewed by a stationary observer. Consequently, in the current study we predicted that performance on a place-learning task may be significantly correlated with memory performance in our arena task.

\section{Method}

\subsection{Participants}

Eighty university students (40 men and 40 women) participated voluntarily in the experiment for research participation credit in undergraduate Psychology classes. The mean age of female participants was 20.0 years (range 18-42 years), and the mean age of male participants was 19.8 years (range 18-26 years). No participants reported visual or motor problems that would have influenced their performance.

\subsection{Design}

A $2 \times 2 \times 3$ mixed factorial design was employed. The between-subjects factors were cue condition, with two levels (two cues or four cues), memory load, with two levels (one target object or three objects), and the within-subjects factor was delay interval, with three levels (immediate, one-week delay, and two-week delay).

Place learning and memory task. The apparatus for the place learning and memory task was a circular arena, $3 \mathrm{~m}$ in diameter and $3 \mathrm{~m}$ in height located inside a $5 \times 7 \mathrm{~m}$ laboratory. The arena was formed by black curtains that had nine equally spaced entry points, which were places where adjacent curtains were not permanently attached to each other. The arena had a ceiling constructed of translucent white cloth that allowed light from four equally spaced light bulbs to illuminate the interior. The floor was covered with wood shavings in which objects could be hidden easily. The curtains, ceiling, and floor provided no reliable basis for specifying locations within the arena. Four pictures (specifically, a tree on the west wall, a heart on the north wall, a cloud on the east wall and a sun on the south wall) or two pictures (the heart on the north wall, and the sun on the south wall) were mounted equidistant from each other on the interior curtains of the arena (see Fig. 1). The pictures were $20 \times 25 \mathrm{~cm}$ in size, and their distance from the floor was approximately $2 \mathrm{~m}$. Locations to be remembered were designated by toy coins $1.5 \mathrm{~cm}$ in diameter and golden in color. The target coin location was on the floor of the arena as shown in Fig. 1, located $42.6^{\circ}$ from the 'tree' cue and $47.4^{\circ}$ from the 'heart' cue, with a radial value of $78.0 \mathrm{~cm}$. In the high-load condition, the two additional coin locations were on the arena floor as shown in Fig. 1. One object was $75.0^{\circ}$ from the 'heart' cue and $15.0^{\circ}$ from the 'cloud' cue, with 
a radial value of $129.4 \mathrm{~cm}$. The other object was $31.0^{\circ}$ away from the 'sun' cue and $59.0^{\circ}$ from the 'cloud' cue, with a radial value of $72.8 \mathrm{~cm}$.

Performance in the place memory task was scored in terms of three measures. First, absolute error served as a measure of metric inaccuracy. It was defined as the distance in $\mathrm{cm}$ from the remembered target location to the actual location. Second, angular bias reflected angular distortion in memory. For this measure, a polar coordinate grid was imposed on the circular arena floor, with radii extending from the center of the arena to the surrounding wall. Angular bias was defined as the signed difference in degrees from the value of the remembered location minus the value of the actual location, with the sign of the angular value indicating the direction of the deviation (negative values represent clockwise bias and positive values represent counterclockwise bias). Third, radial bias was measured in $\mathrm{cm}$ by subtracting the radial distance of the actual point from the radial distance of the observed point. A negative radial value indicates a radial bias toward the center of the circle, whereas a positive value indicates a radial bias toward the circumference of the arena.

Spatial ability tests. Participants completed three spatial tests. Two of the spatial tests, the Maze Learning Test (Allen, Kirasic, Dobson, \& Long, 1996), assessing spatial-sequential memory, and the Money Standardized Road Map Test (Money, 1976), assessing directional sense, were not assumed to relate to the task at hand and merely served as filler tasks. The third task, the Building Memory Test (Ekstrom, French, Harmen, \& Dermen, 1976) was designed to assess spatial-visual memory as a small-place memory task. The objective of the test was to learn the position of buildings in a street map. After studying a map for three minutes, participants were asked to indicate on a test page the location of 12 buildings. The format was multiplechoice, with six possible response choices for each location. Four minutes were allowed for retrieval. The test had two parts, each with a different map and set of 12 test items. Maximum possible score was 24.

\subsection{Procedure}

Each participant was involved in three data collection sessions separated by one-week intervals. The first session involved a learning phase and a test phase. The second and third sessions involved a test phase only.

At the beginning of the first session, the experimenter met the participant outside the room containing the arena, obtained informed consent, and provided instructions. For the learning phase, the participant was seated in a wheelchair and was blindfolded to interfere with the use of visual information outside the arena for orientation. Then the experimenter wheeled the participant to the arena following a circuitous route to reduce the use of vestibular or kinesthetic information for orientation. After wheeling the participant to the first entry point (entry point number 1) the experimenter instructed the participant to remove the blindfold and stand up. Then, inside the arena the experimenter led the participant to the predetermined hiding place(s) and hid the coin(s) under the wood-shavings. The participant was told to remember the hiding place(s) exactly because he or she would later have to indicate the hiding place(s) with a marker. The participant was given as long as needed to memorize the hiding place of the coin(s). No participant needed more than 1 minute. After he or she had 
memorized the hiding place(s), the blindfold was replaced, and the participant was wheeled out from the room containing the arena by a circuitous route. This exit ended the learning phase.

Outside the room, the participant was administered the Building Memory Test as a filler task, followed by the test phase of the arena procedure. The experimenter wheeled the blindfolded participant back into the room containing the arena and, following circuitous routes each time, stopped at three different entry points (entry point number 7,1 , and 5). As during the learning phase, at each entry point the participant was instructed to remove the blindfold and stand. Inside the arena, the participant was instructed to orient himself or herself, walk from the entry point position directly to the hiding place or series of hiding places, and to indicate the hiding place(s) by placing a small red plastic disk to mark the exact location. By instructing the participant to walk directly from the entry point position to the hiding place or series of hiding places, the participant was restricted in his/her use of strategy and could not rely on any physical measurement that he/she might have used during the learning phase. After placing the marker(s), the participant came back to the entry point of that trial, sat in the wheelchair, and replaced the blindfold. The experimenter recorded the responses quickly using a grid in place under the wood shavings. After the third test trial, the experimenter wheeled the participant outside the room containing the arena. This concluded the initial session.

Participants returned one week later for the second session. Initially, they were administered the Maze Learning Test. Then, they were seated in the wheelchair, blindfolded, and conveyed into the room containing the arena. The events comprising the second test phase were the same as those in the first session, except that a different sequence of entry sites was involved (entry point number 1,2, and 6). The second session ended with the participant being wheeled from the room after the third entry point.

Participants returned two weeks after the initial session for the third session. Initially, they were administered the Road Map Test. Then, testing proceeded as in the first and second sessions, except that a different sequence of entry sites was involved (entry point number 8,3 , and 1 ). The third session ended with the participant being wheeled from the room after the third entry point. Participants were then debriefed and thanked for their participation.

\section{Results}

\subsection{Overview of Analyses}

Data from the place memory task were analyzed in a series of analyses of variance (ANOVA's). The primary analyses were performed using as the dependent measures the mean absolute error, angular bias, and radial bias, from the three trials on each session. Sex of participant was not integrated in any of these analyses because preliminary analyses did not reveal any significant effects or interactions involving this variable $(p>.05)$. We report analyses only for the target location, as it was common to all conditions. 
For the variable absolute error and radial bias, if participants had a score of more than two standard deviations above the mean for that condition then the data point was replaced by the mean for that condition. Outlying data points for the variable angular bias were identified by an error of $90^{\circ}$ or greater. This method was used in order to eliminate cases that indicated misplacement into an adjacent quadrant. In the two cue condition, out of 120 data points (40 participants with one mean estimate in each of 3 sessions) 8 data points $(6.7 \%)$ were replaced for absolute error, $6(5 \%)$ for radial bias, and $5(4.2 \%)$ for angular bias. In the four cue condition out of 117 data points (39 participants with one mean estimate in each of 3 sessions) 4 data points $(3.4 \%)$ were replaced for absolute error, $6(5.1 \%)$ for radial bias, and $0(0 \%)$ for angular bias. No effects for trials or memory load were noted regarding outlying data points. For all statistical analyses, the significance level was set at .05.

\subsection{Analyses of Absolute Error (in cm)}

Fig. 2 illustrates how absolute error for the target location varied as a function of delay interval and load. The principal analysis of absolute error was a 2 (Cue Condition) x 2 (Memory Load) x 3 (Delay Interval) mixed ANOVA performed on mean absolute error per session for the target object location. Results indicate a main effect of number of cues, $F(1,75)=4.07, p=.047, M S e=490.11$, reflecting greater accuracy when more peripheral cues were available, $M_{4 \text {-Cues }}=31.21(S D=11.70)$ and $M_{2 \text {-Cues }}=36.98(S D=14.88)$. The analysis also showed a significant main effect of memory load, $F(1,75)=6.52, p=.013, M S e=490.11$, reflecting greater accuracy in the low-load condition, $M_{\text {Low }}=30.44(S D=12.72)$ and $M_{\text {High }}=37.77(S D=13.62)$. The Cue $\mathrm{x}$ Load interaction did not reach conventional levels of significance in our

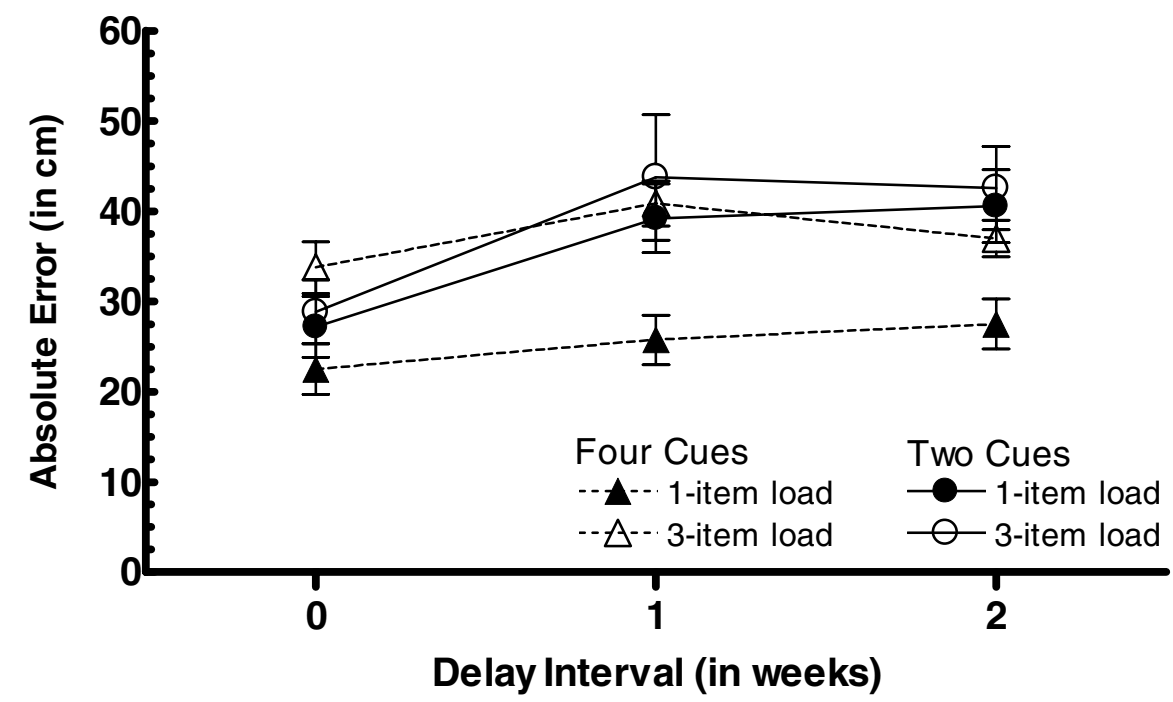

Fig. 2. Mean absolute error $( \pm$ SEM) for the target object location across delay-interval session for each load condition 
analysis ${ }^{1}$. The main effect of delay interval was significant, $F(2,150)=13.99$, $p<.001$. The trend analysis revealed a significant linear trend, $F(1,75)=20.50, p<$ .001 , along with a quadratic trend, $F(1,75)=7.91, p<.01$. Post hoc analyses indicated that participants produced less error when tested immediately after acquisition trial than after a one-week delay $(p<.001)$ or after a two-week delay $(p<$ $.001)$. No difference was noted between a one-week and a two-week delay. There was also a significant Cue x Delay interaction, $F(2,150)=3.40, p=.036, M S e=155.15$, reflecting similar accuracy when tested immediately after acquisition trials, but higher accuracy in the four-cue condition after one and two week delays relative to the two cue condition (combined across load condition).

To test for trial effects, an additional analysis was conducted adding the three trials within each session as a within-subjects factor. Results for a 2 (Cue Condition) x 2 (Memory Load) x 3 (Delay Interval) x 3 (Trial) mixed ANOVA indicated a significant main effect of trials, $F(2,150)=11.41, p<.001, M S e=152.41$, reflecting greater accuracy for the first and second trial, $M=33.29(S D=14.46)$ and $M=32.25$ $(S D=13.62)$, respectively, compared to the third trial with $M=37.06(S D=15.80)$. Further, no significant interactions were noted with trial.

\subsection{Analyses of Angular Bias (in degrees)}

Fig. 3 illustrates how angular bias for the target location varied as a function of delay interval and load. A parallel 2x2x3 ANOVA was conducted on angular bias. Results indicate an overall effect of number of cues, $F(1,75)=13.87, p<.001$, MSe = 1278.77 , reflecting a bias effect in opposite directions for the different cue conditions, $M_{2 \text {-Cues }}=12.55(S D=20.51)$ and $M_{4 \text {-Cues }}=-4.60(S D=21.23)$. This opposite bias effect is consistent with the cue-based fuzzy-boundary prediction of bias toward the nearest cue. The main effect of delay interval was not significant, but there was a significant Cue $\mathrm{x}$ Delay interaction, $F(2,150)=11.06, p<.001, M S e=137.55$. This interaction was due to much stronger bias effects for the one and two week delays than for the no delay condition.

In a more detailed analysis of bias effects, one-sample t-tests were conducted at each interval for each cue and load condition. No significant bias effects were noted in any of the groups when tested immediately after the acquisition trial, indicating good shortterm spatial memory for all four groups. Unbiased memory, with no significant effects at any of the three delays, was further noted when four cues were available and only one target location had to be remembered, indicating stable memory across time. However, in the four-cue high-load condition, memory for the target location was significantly biased in the one-week delay interval toward the nearest available environmental cue $(t$ $=-2.66, p=.015)$. For the two-cue condition, both high and low memory load groups demonstrated significant bias effects toward the nearest available cue for the one-week

\footnotetext{
${ }^{1}$ Although the Cue X Load interaction looks strong in Fig. 2, it was not significant when we conducted analyses on the untransformed scores. However, it was statistically significant when the ANOVA was conduced on the log transformation of the error scores, $F(1,75)=$ $5.16, p=.026$. This interaction reflected the significantly reduced absolute error for the target in the four-cue low-load condition compared to the other three conditions. However, a test of violations of sphericity was significant for the log transformed scores but not for the untransformed scores, so this result must be viewed with caution.
} 
delay $(t=3.45, p=.003$ and $t=2.25, p=.037$, respectively) and for the two-week delay $(t=3.04, p=.007$ and $t=2.89, p=.010$, respectively). Note that all significant tests of bias were in the direction predicted by the cue-based fuzzy-boundary theory (Fitting et al., 2005).

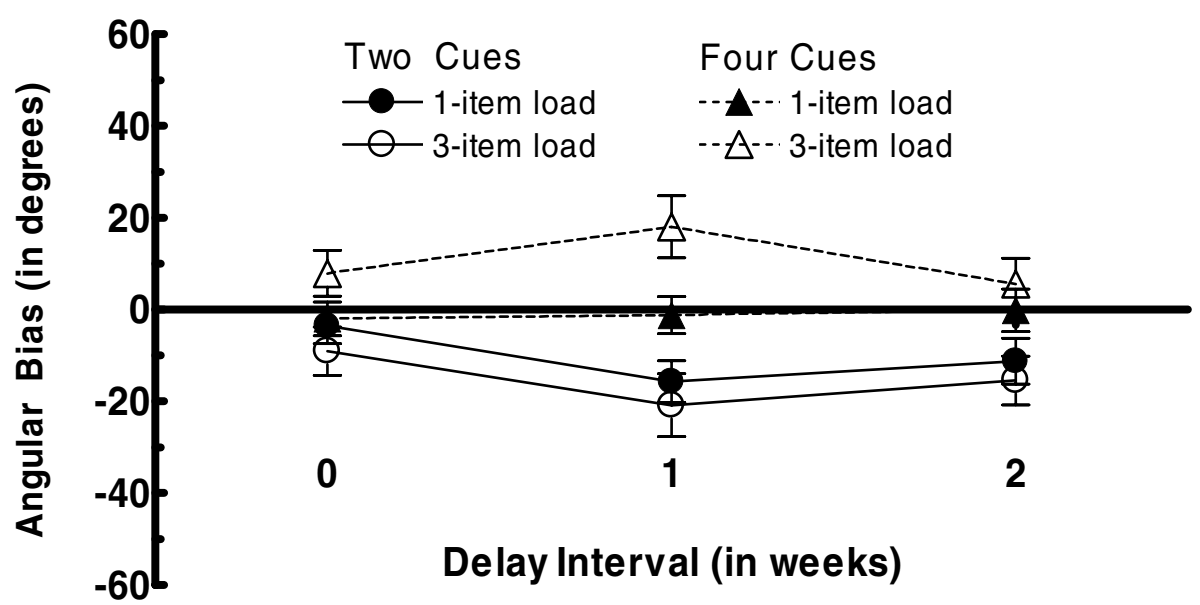

Fig. 3. Mean angular bias $( \pm$ SEM) for the target object location across delay interval session for each load condition

\subsection{Analyses of Radial Bias (in cm)}

Fig. 4 illustrates how radial bias for the target location varied as a function of delay interval and load. A parallel $2 \times 2 \times 3$ ANOVA was performed on mean radial bias. Results indicate an overall load effect, $F(1,75)=16.92, p<.001, \mathrm{MSe}=448.88$, reflecting greater bias toward the circumference in the low-load condition relative to the high-load condition, $M_{\text {Low }}=9.97(S D=12.62)$ and $M_{\text {High }}=-1.43(S D=11.88)$. No other effects reached conventional levels of significance.

\subsection{Correlation Analysis}

Pearson correlations were conducted on the paper and pencil Building Memory Test of spatial location and the three measures from the human arena memory task (after reverse scoring angular bias in the four-cue condition so that positive values in both conditions indicated a bias toward the nearest environmental cue). There were no significant correlations between the small-scale memory task and any of the largescale performance measures. However, correlations between absolute error and angular bias scores were significant for each session and averaged $r=.47, p<.001$. This finding supports our prior predictions that bias is a contributor to error. No significant correlation with absolute error was noted for radial bias. 


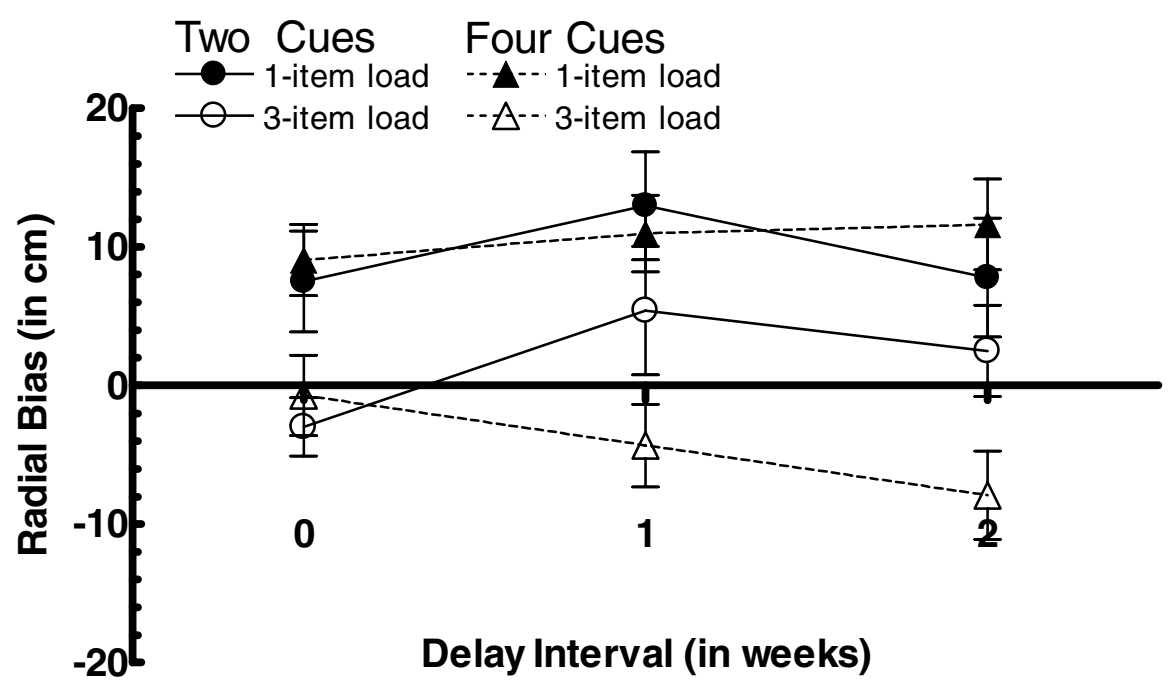

Fig. 4. Mean radial bias $( \pm$ SEM) for the target object location across delay interval session for each load condition

Finally, we investigated the stability of individual differences in the arena task performance by computing Cronbach's alpha across three delay intervals. Cronbach's alpha was 0.74 , indicating relatively stable individual differences in spatial memory. Thus although stable individual differences were found, these were not predicted by the small-scale test.

\section{Conclusion}

We conducted a human analog study of the Morris Water Maze using a traversable arena. It is important to note that our version of the Water Maze analog differed from the typical Water Maze procedure and human analog versions of that procedure used in the past in several ways. First, in our procedure there was only one learning trial, which consisted of the individual freely exploring the area and considering ways to remember the exposed target. In the typical procedure, the target is hidden and the individual initially finds it through free exploration, and then additional learning trials are recorded. Second, animal studies and human studies, such as those reported by Leplow et al. (2003), typically track the path of the individual searching for the hidden object so that dependent variables are based on the movement record, recording proximity to the target, movement away from the target, time to find the target and other process measures. These measures are related to a third fundamental difference between our study and the classic Water Maze procedure, which is that in our study the individual does not receive feedback on the location of hidden target; in the classic procedure such feedback is given when the individual finds the target. These differences arose from our attempt to blend aspects of the small scale place memory paradigm of Huttenlocher et al. (1991) with the navigation-based procedure 
of the Water Maze. Our design then allowed us to use dependent measures more suitable for assessing memory representation of the location of the target and associated biases.

In our study, we manipulated three main variables: number of cues, number of targets (or load), and delay interval. Manipulation of these variables provided us with the tools to assess basic memory performance in this traversable and mostly undifferentiated space. Not surprisingly, memory accuracy (inversely related to absolute error) decreased with increase in delay interval, increase in number of targets to remember, and decrease in number of cues. Despite decrements in performance related to these variables, the overall performance of participants demonstrated robust memory for spatial location. Even after a two week delay with no feedback, participants placed the target on average within about $37.7 \mathrm{~cm}$ of the actual target location (inside the $300 \mathrm{~cm}$ diameter task field). In the most advantageous of task conditions (one-item to be remembered using four cues), the target was placed within about $27.3 \mathrm{~cm}$ of its true location after a two week delay. The forgetting curves, linear with a minor quadratic component, indicate that most of the forgetting is within the first week, as there was no significant increase in absolute error from week 1 to week 2. Thus, within this task our subjects were capable of accurate spatial memory after a minimal encoding session.

The lack of performance differences between one and two week delays is subject to different interpretations. One possibility is that forgetting asymptotes after one week, as indicated in this study. Alternatively, the one-week trial may have served as a retrieval and rehearsal opportunity that bolstered memory for the hidden coin location(s) and thus obscured any additional forgetting that may have occurred. A research design that manipulates delay between subjects so that participants have no intermediate retrieval trials would address this issue. However, the advantage of the reported experiment which utilized a within-subjects manipulation of delay is that it provided a much more powerful test of the delay factor than a comparable betweensubjects design.

An important innovation of the current study as compared with previous human analog studies of the Morris Water Maze was our focus on predicting bias in spatial memory. We tested whether theories of spatial memory bias established in a small two-dimensional space (Huttenlocher et al., 1991; Fitting et al., 2005) would translate to the larger traversable space in our arena apparatus. In particular, we tested predictions generated from our cue-based fuzzy-boundary model of spatial memory (Fitting et al., 2005). These predictions were supported in three ways. First, angular bias was in the predicted direction: The remembered location shifted toward the heart cue in the two-cue condition and away from the heart cue (toward the tree cue) in the four-cue condition. This is consistent with cues being used as category prototypes and uncertainty about location being resolved toward the prototype, as proposed by the category-adjustment model (Huttenlocher et al., 1991). A second way in which model predictions were supported was the increase in angular bias and absolute error for the two-cue versus the four-cue condition. The cue-based fuzzy trace model predicts that error and bias increase as distance to the nearest adjacent cues increase (Fitting et al., 2005). Adjacent cues were more closely spaced in the four-cue condition and so error was reduced. A third way in which the model was supported was in the relationship between error and bias. The model predicts that bias will increase as memory errors 
increase. Thus, the fact that bias was only significant at the 1 and 2 week delay intervals in which absolute error was greatest is consistent with the model. Furthermore, absolute error significantly correlated with predicted bias, consistent with the idea that the greater the error in memory, the more likely the error will be resolved in a biased direction.

Although some small effects on radial bias were observed, these were not the focus of the investigation. The significant load effect on radial bias is consistent with the radial prototype being influenced by the existence of other radial locations, possibly averaging across the radial locations in order to form a radial prototype. Such speculation would need to be systematically tested in future research.

The correlational analyses indicated stable individual differences in our arena task. These were not related to sex differences and were not predicted by a small-scale paper and pencil spatial memory task. Thus, one area of future investigation would be to determine what small-scale tasks predict performance in the larger-scale spatial memory task.

In summary, the present study provided evidence for the impact of the three investigated basic factors on human place memory: environmental cue availability, delay interval, and memory load. The most important implication of the present research is that place memory in a large-scale situation may be governed by an imposed coordinate system, with errors resolved toward the nearest available peripheral cue in the environment, as demonstrated in previous small-scale tasks (Fitting, 2005). Although further investigation using a variety of cue and spatial locations is needed, these results support the idea that cues are used as prototypes, with memory for locations biased toward the nearest available environmental cue and the magnitude of bias being a function of distance to the available cue. While the test environment used in our study was traversable, its size was necessarily small and comparable to that used in other Water Maze analog tasks (Leplow, Höll, Zeng, \& Mehdorn, 1998). As such, our test area would be considered a vista space (Montello, 1993), which may not necessarily afford active exploration for building a representation. However, we believe this study provides the groundwork for future tests of bias in large scale, traversable environments.

Acknowledgement. The authors gratefully thank Lena Fitting for helping with the data transformation.

\section{References}

Allen, G.L., Kirasic, K., Dobson, S.H., Long, R.G.: Predicting environmental learning from spatial abilities: An indirect route. Intelligence 22, 327-355 (1996)

Astur, R.S., Ortiz, M.L., Sutherland, R.J.: A characterization of performance by men and women in a virtual Morris water task: A large and reliable sex difference. Behavioural Brain Research 93, 185-190 (1998)

Astur, R.S., Taylor, L.B., Mamelak, A.N., Philpott, L., Sutherland, R.J.: Humans with hippocampus damage display severe spatial memory impairments in a virtual Morris water task. Behavioural Brain Research 132, 77-84 (2002)

Cheng, K.: A purely geometric module in the rat's spatial representation. Cognition 23(2), 149178 (1986) 
Dent, K., Smyth, M.M.: Verbal coding and the storage of form-position associations in visualspatial short-term memory. Acta Psychologica 120, 113-140 (2005)

Devan, B.D., Goad, E.H., Petri, H.L.: Dissociation of hippocampal and striatal contributions to spatial navigation in the water maze. Neurobiology of Learning and Memory 66, 305-323 (1996)

Egan, D.: Testing based on understanding: Implications from studies of spatial ability. Intelligence 3, 1-15 (1979)

Ekstrom, R.B, French, J.W., Harmen, H.H., Dermen, D.: Manual for kit of factor-referenced cognitive tests. Educational Testing Service, Princeton, NJ (1976)

Fenton, A.A., Arolfo, M.P., Nerad, L., Bures, J.: Place navigation in the Morris water maze under minimum and redundant extra-maze-cue conditions. Behavioral and Neural Biology 62, 178-189 (1994)

Fitting, S.: Memory for spatial location: Cue effects as a function of field rotation. Master's Thesis, Department of Psychology, University of South Carolina, Columbia, SC (2005)

Fitting, S., Wedell, D.H., Allen, G.L.: Memory for spatial location: Influences of environmental cues and task field rotation. In: Cohn, A.G., Mark, D.M. (eds.) COSIT 2005. LNCS, vol. 3693, pp. 459-474. Springer, Heidelberg (2005)

Gallistel, C.R.: The organization of learning. MIT Press, Cambridge (1990)

Grön, G., Wunderlich, A.P., Spitzer, M., Tomczak, R., Riepe, M.W.: Brain activation during human navigation: gender-different neural networks as substrate of performance. Nature Neuroscience 3, 404-408 (2000)

Haun, D.B.M., Allen, G.L., Wedell, D.H.: Bias in spatial memory: a categorical endorsement. Acta Psychologica 118, 149-170 (2005)

Hebb, D.O.: The organization of behaviour. Wiley-Interscience, New York (1949)

Hermer, L., Spelke, E.S.: A geometric process for spatial reorientation in young children. Nature 370(6484), 57-59 (1994)

Huttenlocher, J., Hedges, L.V., Corrigan, B., Crawford, L.E.: Spatial categories and the estimation of location. Cognition 93, 75-97 (2004)

Huttenlocher, J., Hedges, L.V., Duncan, S.: Categories and particulars: Prototype effects in estimating spatial location. Psychological Review 98, 352-376 (1991)

Jacobs, W.J., Thomas, K.G.F., Laurance, H.E., Nadel, L.: Place learning in virtual space II. Topographical relations as one dimension of stimulus control. Learning and Motivation 29, 288-308 (1998)

Jarrard, L.E.: On the role of the hippocampus in learning and memory in the rat. Behavioral and Neural Biology 60, 9-26 (1993)

Knierim, J.J., Rao, G.: Distal landmarks and hippocampal place cells: effects of relative translation versus rotation. Hippocampus 13(5), 604-617 (2003)

Lehnung, M., Leplow, B., Ekroll, V., Herzog, A., Mehdorn, M., Ferstl, R.: The role of locomotion in the acquisition and transfer of spatial knowledge in children. Scandinavian Journal of Psychology 44, 79-86 (2003)

Leplow, B., Höll, D., Zeng, L., Mehdorn, M.: Spatial orientation and spatial memory within a 'locomotor maze' for humans. In: Freska, C., Habel, C., Wender, K.F. (eds.) Spatial Cognition. LNCS (LNAI), vol. 1404, pp. 429-446. Springer, Heidelberg (1998)

Leplow, B., Lehnung, M., Pohl, J., Herzog, A., Ferstl, R., Mehdorn, M.: Navigational place learning in children and young adults as assessed with a standardized locomotor search task. British Journal of Psycholog 94, 299-317 (2003)

Money, J.: Manual: A standardized road-map test of direction sense. Academic Therapy Publications, San Rafael, California (1976) 
Montello, D.R.: Scale and multiple psychologies of space. In: Frank, A.U., Campari, I. (eds.) COSIT 1993. LNCS, vol. 716, pp. 312-321. Springer, Heidelberg (1993)

Morris, R.G.M.: Spatial localization does not require the presence of local cues. Learning and Motivation 12, 239-260 (1981)

Morris, R.G.M., Garrud, P., Rawlins, J.N.P., O'Keefe, J.: Place navigation impaired in rats with hippocampal lesions. Nature 297, 681-683 (1982)

Morris, R.G., Parslow, D.M.: Neurocognitive components of spatial memory. In: Allen, G.L. (ed.) Human Spatial Memory: Remembering where, pp. 217-247. Lawrence Erlbaum Associates, Inc, London (2004)

Nadel, L.: Varieties of spatial cognition: Psychobiological considerations. In: Diamond, A. (ed.) Annals of the New York Academy of Sciences, vol. 608, pp. 613-636. Academy of Sciences, New York (1990)

Newcombe, N.S., Huttenlocher, J.: Making space: The developmental of spatial representation and spatial reasoning. MIT Press, Cambridge, MA (2000)

Overman, W.H., Pate, B.J., Moore, K., Peuster, A.: Ontogeny of place learning in children as measured in the radial arm maze, Morris search task, and open field task. Behavioral Neuroscience 110(6), 1205-1228 (1996)

O'Keefe, J., Nadel, L.: The hippocampus as a cognitive map. Clarendon Press, Oxford (1978)

Parslow, D.M., Morris, R.G., Fleminger, S., Rahman, O., Abrahams, S., Recce, M.: Allocentric spatal memory in humans with hippocampal lesions. Acta Psychologica 118(1-2), 123-147 (2005)

Siegel, A.W., White, S.H.: The development of spatial representations of large-scale environments. In: Reese, H.W. (ed.) Advances in child development and behavior, vol. 10, pp. 9-55. Academic Press, New York (1975)

Sutherland, R.J., Rudy, J.W.: Configural association theory: The role of the hippocampal formation in learning, memory, and amnesia. Psychobiology 17, 129-144 (1987)

Tolman, E.C.: Cognitive maps in rats and men. Psychological Review 55, 189-208 (1948)

Wedell, D.H., Fitting, S., Allen, G.L.: Shape effects on memory for location. Psychonomic Bulletin \& Review (in press) 